\title{
The Influence of Phlorotannins and Bromophenols on the Feeding Behavior of Marine Herbivorous Gastropod Turbo cornutus
}

\author{
Toshiyuki Shibata $^{1 *}$, Taiko Miyasaki ${ }^{2}$, Hideo Miyake ${ }^{1}$, Reiji Tanaka $^{1}$, Shigeo Kawaguchi ${ }^{3}$ \\ ${ }^{1}$ Major of Life Sciences, Graduate School of Bioresources, Mie University, Mie, Japan; ${ }^{2}$ Department of Food Science and Technolo- \\ gy, National Fisheries University, Yamaguchi, Japan; ${ }^{3}$ Faculty of Agriculture, Kyushu University, Fukuoka, Japan. \\ Email: "shibata@bio.mie-u.ac.jp
}

Received December $6^{\text {th }}, 2013$; revised January $16^{\text {th }}, 2014$; accepted January $28^{\text {th }}, 2014$

Copyright (c) 2014 Toshiyuki Shibata et al. This is an open access article distributed under the Creative Commons Attribution License, which permits unrestricted use, distribution, and reproduction in any medium, provided the original work is properly cited. In accordance of the Creative Commons Attribution License all Copyrights (C) 2014 are reserved for SCIRP and the owner of the intellectual property Toshiyuki Shibata et al. All Copyright (C) 2014 are guarded by law and by SCIRP as a guardian.

\section{ABSTRACT}

The influence of phlorotannins and bromophenols on the feeding behavior of marine herbivores was determined using bioassay of Turbo cornutus. It was found that phloroglucinol and its oligomers isolated from the brown alga Eisenia bicyclis (eckol, fucofuroeckol A, phlorofucofuroeckol A, dieckol, and 8,8'-bieckol) had a deterrent effect against feeding behavior of $T$. cornutus in the concentration of algal body, respectively. In the case of the examination of $0.1 \mathrm{mM}$ concentration, although fucofuroeckol A and phlorofucofuroeckol A significantly reduced feeding by $T$. cornutus, phloroglucinol and 8,8'-bieckol did not show any significant influence on feeding behavior, and eckol and dieckol had stimulating activity. 2,4-Dibromophenol and 2,4,6-tribromophenol, which are major components of extracellular secretions from Eisenia and Ecklonia species, caused the death, the torpidity, and the decreased appetite of $T$. cornutus at the concentration of $0.1 \mathrm{mM}$. In addition, 2,4-dibromophenol had strong feeding deterrent activity at the concentration of $1 \mu \mathrm{M}$. These results indicate that phlorotannins and bromophenols act as chemical defense agents of brown algae against environmental stresses such as the herbivore attack.

\section{KEYWORDS}

Bromophenols; Brown Algae; Chemical Defenses; Feeding Deterrent; Eisenia bicyclis; Phlorotannins; Plant-Herbivore Interactions; Turban Shell; Turbo cornutus

\section{Introduction}

Phlorotannins of marine algal polyphenols, which have been found exclusively in brown algae, are formed by the polymerization of phloroglucinol (1,3,5-trihydroxybenzene) $[1,2]$. In marine chemical ecology, phlorotannins (as well as tannins from terrestrial plants) are considered to be an important component of the chemical defense material against natural enemies (e.g., marine herbivores, bacteria, epiphytes and ultraviolet). Many investigators have discussed the biological activities and roles of phlorotannins [1-7]. To our knowledge, however, most of

\footnotetext{
*Corresponding author.
}

the reports on the defensive function of phlorotannins present no findings regarding a "relationship between chemical structures of the compounds and biological activities", "correlation of the activities and concentration of phlorotannins" and "evaluation of the activities based on actual concentration of the compounds in the algal body”.

Previously, we isolated the phlorotannins, these being eckol (a phloroglucinol trimer), fucofuroeckol A (a tetramer), phlorofucofuroeckol A (a pentamer), dieckol and 8,8'-bieckol (hexamers) from Japanese Eisenia and Ecklonia species and reported their chemical and local distribution in the algae [8]. The brown algae are distrib- 
uted along the temperate coasts of Japan and form a kelp bed called a "marine forest" in the subtidal zone [9]. In the preceding reports [10-12], the following two facts became apparent: 1) the phenolic substances secreted from the living brown algae consisted of the two monomeric bromophenols, 2,4-dibromophenol and 2,4,6-tribromophenol; 2) phloroglucinol and phlorotannins were kept strictly within the algal body, whilst the algae were alive. It is indicated that extracellular phenolic substances of living brown algae have some deterrent activities against natural enemies [1,13-15]. Though it has been thought that the substances are phlorotannins in many past reports [1,13-15], the monomeric bromophenols may play a deterrent role against natural enemies such as marine herbivores.

In this study, we isolated the phlorotannins from Eisenia bicyclis and determined the influence of each compound on feeding behavior using a bioassay of marine herbivorous gastropod T. cornutus. Then, the influence of bromophenols on their feeding behavior was evaluated. The mechanism of chemical defense via phenolic compounds in the brown algae was also discussed.

\section{Materials and Methods}

\subsection{Materials}

The brown alga E. bicyclis (Kjellman) Setchell, without any visible grazing or other tissue damage, was collected from the coasts of the Itoshima Peninsula $\left(33^{\circ} 37^{\prime} \mathrm{N}\right.$, $\left.130^{\circ} 10^{\prime} \mathrm{E}\right)$ in Fukuoka Prefecture, Japan. The alga was washed with filtered seawater, air-dried, and pulverized. The algal powder was stored at $-30^{\circ} \mathrm{C}$ until use.

T. cornutus (approx., $6.5 \mathrm{~cm}$ in shell length) were purchased from the Fisheries Cooperative Association of Yoshimi in Yamaguchi prefecture, Japan. 2,4-Dibromophenol and 2,4,6-tribromophenol were purchased from Wako Pure Chemicals (Osaka).

\subsection{Extraction and Purification of Phlorotannins}

Extraction of the phlorotannins from the algal powder was carried out according to the method described in previous reports [11,16,17]. Each of the phlorotannins in the crude extracts was partially purified on a silicic acid column according to the same method described in a previous report [16]. Further purification of the phlorotannins was carried out using preparative HPLC system. The HPLC system consisted of LC-6AD pumps (Shimadzu, Japan), a CBM-20A system controller (Shimadzu, Japan), a SPD-20A UV detector (Shimadzu, Japan) and an Inertsil ODS-3 column (10 mm I.D. × $250 \mathrm{~mm}$, GL Science, Japan). Elution was performed at a flow-rate of $4.7 \mathrm{~mL} / \mathrm{min}$ with a linear gradient from $30 \%$ to $100 \%$
$\mathrm{MeOH}$ for $20 \mathrm{~min}$, and maintained for an additional 20 min. The UV detector was set at $290 \mathrm{~nm}$. The purity was confirmed by three-dimensional HPLC using a photodiode array detector (SPD-M10AV, Shimadzu, Japan) with an Inertsil ODS-3 column (4.6 mm I.D. × 250 mm, GL Science, Japan) $[11,16]$. The identification of the purified phlorotannins was carried out using an LC/MS system (6120 Quadrupole LC/MS with 1260 Series HPLC System, Agilent, CA, USA) with an Inertsil ODS-3 column (4.6 mm I.D. $\times 150 \mathrm{~mm}$, GL Science, Japan). Elution was performed at a flow-rate of $0.5 \mathrm{~mL} / \mathrm{min}$ with a linear gradient from $30 \%$ to $100 \% \mathrm{MeOH}$ for $20 \mathrm{~min}$, and maintained for an additional 20 min. Each phlorotannin was monitored by ESI-MS/APCI-MS multimode analysis in positive and negative mode.

\subsection{Bioassays of Feeding Behavior of the Marine Herbivorous Gastropod, T. cornutus}

The bioassays of feeding behavior were carried out according to the method of Miyasaki and Harada [18]. The experiment herbivore, $T$. cornutus was fed with the dead brown alga E. bicyclis, as its daily feed. The experiment food media were prepared as follows: first, each of the phenolic compounds dissolved in $\mathrm{MeOH}$ was mixed with dextran. After removal of the $\mathrm{MeOH}$ with a lyophilizer, the mixture was homogenized and this was then dissolved in $40 \mathrm{~mL}$ of a $2 \%$ agar medium in a Petri dish $(9.0$ $\mathrm{cm}$ i.d. $\times 8.0 \mathrm{~mm}$ ). Control medium without the phenolic compounds was also prepared. Each of the experimental and control media was placed in pairs in an aquarium $(270$ w. $\times 350$ d. $\times 130$ h. mm) with running seawater $(150 \mathrm{~mL} / \mathrm{min})$ and aeration. T. cornutus (8 individuals/aquarium) was allowed to feed for $16 \mathrm{~h}$ after sunset. Finally, after the bioassay of the feeding, the amount of media consumed was weighed. The data are presented as mean \pm standard deviation of analytical values of independent tests $(n=10)$. The statistical analysis of the data was performed using $t$-test $(P<0.05)$.

All reagents used in this experiment were of analytical grade.

\section{Results and Discussion}

It is shown that the crude extracts from the brown algae including phlorotannins have a feeding deterrent activity against marine herbivores in the past reports $[1,3,5,6]$. The aim of this study was mainly to clarify the relationship between the chemical structures of phlorotannins and their influence on the feeding of marine herbivores. First, to evaluate the function of each phlorotannins, the compounds were isolated from the E. bicyclis. Figure 1 shows the chemical structures of phlorotannins used in this study. These phlorotannins were refined to a purity 


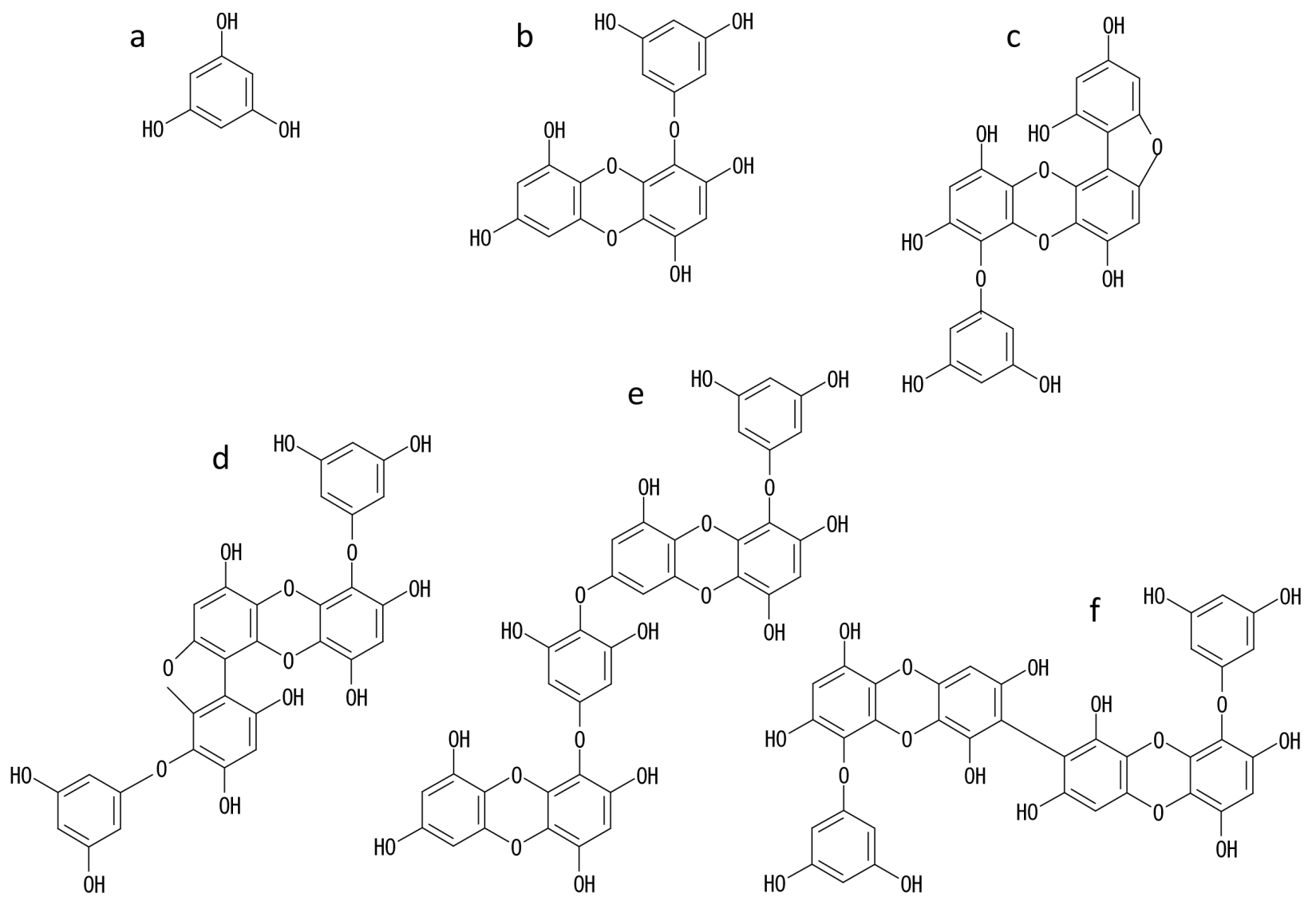

Figure 1. Chemical structures of phloroglucinol and phlorotannins isolated from the brown alga $E$. bicyclis. These compounds were isolated from crude phlorotannins of $E$. bicyclis by column chromatography and preparative HPLC. (a) phloroglucinol; (b) eckol; (c) fucofuroeckol A; (d) phlorofucofuroeckol A; (e) dieckol; (f) 8,8'-bieckol.

of $98 \%$ or more via column chromatography and preparative HPLC (data not shown). In the previous paper [8], it was revealed that the yield of total phlorotannins among Japanese Eisenia and Ecklonia species was about $3.0 \%$ of the algal powder (dry weight) and the content and composition of the phlorotannins did not vary seasonally. Therefore, based on these findings, each of the phloroglucinol and isolated phlorotannins, which was equivalent to the natural concentration in an algal body, was added to the experiment food medium and used to the bioassay using marine herbivorous gastropod $T$. cornutus (Table 1). As a result, without any relation to extent polymerization of phloroglucinol, it is found that all examined compounds have deterrent activity against feeding behavior of $T$. cornutus in the concentration of algal body (Table 1). Under the condition of certain concentration, the influence that each compound exerted on the feeding behavior of $T$. cornutus was also examined (Table 2). As a result of comparative test, fucofuroeckol A and phlorofucofuroeckol A significantly deterred feeding by $T$. cornutus at the concentration of 0.1 $\mathrm{mM}$. On the other hand, eckol and dieckol have a stimu- lating effect on feeding activity at the concentration of $0.1 \mathrm{mM}$ and the opposite results were shown (Table 2). It was found that phloroglucinol and 8,8'-bieckol did not have significant influence on feeding behavior (Table 2).

2,4-Dibromophenol and 2,4,6-tribromophenol, which are halo metabolites, are major components of extracellular secretions from Eisenia and Ecklonia species $[11,12]$. Then, an examination of the influence of these bromophenols on the feeding behavior of $T$. cornutus was conducted. In order to compare with the data of phlorotannins shown in Table 2, the effect of each bromophenol was measured at the concentration of $0.1 \mathrm{mM}$. As a result, both monomeric bromophenols caused the death, the torpidity, and the decreased appetite of T. cornutus (data not shown). Therefore, it was found that 2,4-dibromophenol and 2,4,6-tribromophenol demonstrated deterrent activity much stronger than that of phlorotannins at the concentration of $0.1 \mathrm{mM}$. Table 3 shows the effect of each bromophenol when the concentration in a medium is $1 \mu \mathrm{M}$ or less. At the concentration of $1 \mu \mathrm{M}$, although 2,4-dibromophenol had a strong deterrent effect on feeding activity, 2,4,6-tribromophenol 
Table 1. Influence of phloroglucinol and phlorotannins on feeding behavior of marine herbivorous gastropod T. cornutus.

\begin{tabular}{|c|c|c|c|c|}
\hline \multirow{2}{*}{ Compounds $^{* *}$} & \multirow{2}{*}{ Concentration (mM) } & \multicolumn{2}{|c|}{ Amount of media consumed (g) } & \multirow{2}{*}{ Relative to control (A/C) } \\
\hline & & Addition (A) & Control (C) & \\
\hline Phloroglucinol & 0.444 & $15.4 \pm 9.2^{*}$ & $26.1 \pm 8.9$ & 0.59 \\
\hline Eckol & 1.410 & $13.4 \pm 5.2^{*}$ & $19.8 \pm 6.9$ & 0.68 \\
\hline Fucofuroeckol A & 0.615 & $4.5 \pm 1.2^{*}$ & $9.1 \pm 0.9$ & 0.49 \\
\hline Phlorofucofuroeckol A & 2.440 & $14.4 \pm 5.7^{*}$ & $23.8 \pm 7.5$ & 0.61 \\
\hline Dieckol & 2.170 & $11.7 \pm 6.7^{*}$ & $22.6 \pm 6.7$ & 0.52 \\
\hline 8,8'-Bieckol & 1.600 & $9.4 \pm 5.8^{*}$ & $23.5 \pm 6.8$ & 0.40 \\
\hline
\end{tabular}

${ }^{*} P<0.05 .{ }^{* *}$ The amount of each compounds added to the agar, based on the concentration in the brown alga E. bicyclis (Shibata et al., 2004). The data are presented as mean \pm standard deviation of analytical values of independent tests $(n=10)$.

Table 2. Influence of phloroglucinol and phlorotannins on feeding behavior of $T$. cornutus at the concentration of $0.1 \mathrm{mM}$.

\begin{tabular}{cccc}
\hline \multirow{2}{*}{ Compounds } & \multicolumn{2}{c}{ Amount of media consumed (g) } & \multirow{2}{*}{ Relative to control (A/C) } \\
\cline { 2 - 3 } & Addition (A) & Control (C) & 0.84 \\
\hline Phloroglucinol & $11.2 \pm 9.0$ & $13.3 \pm 8.9$ & 1.57 \\
Eckol & $11.8 \pm 5.5^{*}$ & $7.5 \pm 4.5$ & 0.64 \\
Fucofuroeckol A & $10.9 \pm 5.6^{*}$ & $16.9 \pm 5.4$ & 0.69 \\
Phlorofucofuroeckol A & $12.2 \pm 5.8^{*}$ & $17.7 \pm 6.9$ & 1.81 \\
Dieckol & $13.0 \pm 2.9^{*}$ & $7.2 \pm 2.9$ & 0.77 \\
8,8'-Bieckol & $13.3 \pm 4.3$ & $17.3 \pm 7.8$ & \\
\hline
\end{tabular}

${ }^{*} P<0.05$. The data are presented as mean \pm standard deviation of analytical values of independent tests $(n=10)$.

Table 3. Influence of bromophenols on feeding behavior of $T$. cornutus.

\begin{tabular}{|c|c|c|c|c|}
\hline \multirow{2}{*}{ Compounds } & \multirow{2}{*}{ Concentration } & \multicolumn{2}{|c|}{ Amount of media consumed (g) } & \multirow{2}{*}{ Relative to control (A/C) } \\
\hline & & Addition (A) & Control (C) & \\
\hline \multirow[t]{3}{*}{ 2,4-Dibromophenol } & $1 \mu \mathrm{M}$ & $3.9 \pm 3.2^{*}$ & $6.6 \pm 7.2$ & 0.59 \\
\hline & $1 \mathrm{nM}$ & $14.0 \pm 6.9$ & $11.3 \pm 3.3$ & 1.24 \\
\hline & $1 \mathrm{pM}$ & $19.3 \pm 6.1^{*}$ & $11.8 \pm 4.7$ & 1.64 \\
\hline \multirow[t]{5}{*}{ 2,4,6-Tribromophenol } & $1 \mu \mathrm{M}$ & $6.8 \pm 1.7$ & $8.7 \pm 2.9$ & 0.78 \\
\hline & $10 \mathrm{nM}$ & $7.3 \pm 1.8$ & $7.9 \pm 3.3$ & 0.92 \\
\hline & $1 \mathrm{nM}$ & $6.4 \pm 2.2$ & $7.6 \pm 2.6$ & 0.84 \\
\hline & $0.1 \mathrm{nM}$ & $14.6 \pm 4.8$ & $11.7 \pm 6.0$ & 1.25 \\
\hline & $1 \mathrm{pM}$ & $15.6 \pm 7.7$ & $16.9 \pm 6.6$ & 0.92 \\
\hline
\end{tabular}

${ }^{*} P<0.05$. The data are presented as mean \pm standard deviation of analytical values of independent tests $(n=10)$.

was not shown. In addition, it was found that $1 \mathrm{pM}$ 2,4-dibromophenol stimulates predation action of $T$. cornutus (Table 3). At other concentrations, a difference was not seen between the consumption of a sample medium and control medium in either of the bromophenols (Table 3).

In this study, it demonstrated that phloroglucinol and all the examined phlorotannins at concentrations which exist in algal bodies, had feeding deterrent activity against turban shell T. cornutus, and furthermore, bromophenols, in particular 2,4-dibromophenol, were the strong feeding deterrent reagents. On the other hand, it was suggested that phenolic compounds of low concentration (eckol, dieckol and 2,4-dibromophenol) have the possibility of stimulating feeding behavior to T. cornutus. Previously, we reported that phlorotannins showed inhibitory activity of glycosidases present in the viscera of the T. cornutus [10]. Thus, it was suggested that phlorotannins are effective chemical defense agents with the functions of digestive enzyme inhibitor and feeding activity deterrent. Bromophenols are phenols that exist in many algal species [19] and their contents are especially high in red algae and brown algae $[19,20]$. In the previous report [12], we demonstrated that the secretion of 
bromophenols was active in the young plants of species Eisenia and Ecklonia, and their cumulative amount was equivalent to $2.2 \mathrm{mg}$ 2.4-dibromophenol/g wet weight of the plant after $48 \mathrm{~h}$ cultivation. Agatsuma [21] and $\mathrm{Li}$ [22] reported that 2,4-dibromophenol and 2,4,6-tribromophenol had inhibition of larval survival and metamorphosis of the sea urchin. The sea urchin is a predator of marine algae as well as $T$. cornutus. The $T$. cornutus is a representative marine herbivorous gastropod along the Japanese coast. It is well known that the T. cornutus prefers eating dead and/or aged Eisenia and Ecklonia species rather than live ones. From these findings and the present study, it can be considered that the secretion of bromophenols is greatly advantageous for the survival of brown algae. In conclusion, brown algae produce two types of phenolic compounds, storage phenolic compounds comprising phlorotannins, and secretory phenolic compounds comprising bromophenols, and the compounds act as chemical defense agents of brown algae against environmental stress such as herbivore attack.

\section{Acknowledgements}

We acknowledge that the English was revised by Mr. Andrew Rother. This research was supported by Japan Science and Technology Agency, CREST.

\section{REFERENCES}

[1] M. A. Ragan and K.-W. Glombitza, "Phlorotannins, Brown Algal Polyphenols,” In: F. E. Round and D. J. Chapman, Eds., Progress in Phycological Research, Biopress, Bristol, Vol. 4, 1986, pp. 129-241.

[2] C. D. Amsler and V. A. Fairhead, "Defensive and Sensory Chemical Ecology of Brown Algae,” In: J. A. Callow, Ed., Advances in Botanical Research, Elsevier, Amsterdam, Vol. 43, 2006, pp. 1-91.

http://dx.doi.org/10.1016/S0065-2296(05)43001-3

[3] J. A. Geiselman and O. J. McConell, "Polyphenols in Brown Algae Fucus vesiculosus and Ascophyllum nodosum: Chemical Defenses against the Marine Herbivorous Snail, Littorina littorea,” Journal of Chemical Ecology, Vol. 7, No. 6, 1981, pp. 1115-1133. http://dx.doi.org/10.1007/BF00987632

[4] P. D. Steinberg, "Algal Chemical Defense against Herbivores: Allocation of Phenolic Compounds in the Kelp Alaria marginata," Science, Vol. 223, No. 4634, 1984, pp. 405-407. http://dx.doi.org/10.1126/science.223.4634.405

[5] K. Taniguchi, Y. Akimoto, K. Kurata and M. Suzuki, “Chemical Defense Mechanism of the Brown Alga Eisenia bicyclis against Marine Herbivores,” Nippon Suisan Gakkaishi, Vol. 58, No. 3, 1992, pp. 571-575. http://dx.doi.org/10.2331/suisan.58.571

[6] K. Taniguchi, K. Kurata and M. Suzuki, "Feeding-Deterrent Activity of Some Laminariaceous Brown Algae against the Ezo-abalone,” Nippon Suisan Gakkaishi, Vol.
58, No. 3, 1992, pp. 577-581.

http://dx.doi.org/10.2331/suisan.58.577

[7] N. M. Targett and T. M. Arnold, "Predicting the Effects of Brown Algal Phlorotannins on Marine Herbivores in Tropical and Temperate Oceans," Journal of Phycolgy, Vol. 34, No. 2, 1998, pp. 195-205. http://dx.doi.org/10.1046/j.1529-8817.1998.340195.x

[8] T. Shibata, S. Kawaguchi, Y. Hama, M. Inagaki, K. Yamaguchi and T. Nakamura, "Local and Chemical Distribution of Phlorotannins in Brown Algae," Journal of Applied Phycology, Vol. 16, No. 4, 2004, pp. 291-296. http://dx.doi.org/10.1023/B:JAPH.0000047781.24993.0a

[9] T. Yoshida, “Marine Algae of Japan,” Uchida Rokakuho Publishing, Tokyo, 1988.

[10] T. Shibata, K. Yamaguchi, K. Nagayama, S. Kawaguchi and T. Nakamura, "Inhibitory Activity of Brown Algal Phlorotannins against Glycosidases from the Viscera of the Turban Shell Turbo cornutus," European Journal of Phycology, Vol. 37, No. 4, 2002, pp. 493-500. http://dx.doi.org/10.1017/S0967026202003918

[11] T. Shibata, Y. Hama, T. Miyasaki, M. Ito and T. Nakamura, "Extracellular Secretion of Phenolic Substances from Living Brown Algae,” Journal of Applied Phycology, Vol. 18, No. 6, 2006, pp. 787-794. http://dx.doi.org/10.1007/s10811-006-9094-y

[12] T. Shibata, Y. Tashiro and Y. Hama, "Extracellular Secretion of Phenolic Substances from Living Brown Algae: Phlorotannins Are Not Secreted from the Algae into the Seawater Medium," Annual Report Venture Business Laboratory Saga University, Vol. 5, 2004, pp. 89-94.

[13] J. McN. Sieburth and J. T. Conover, "Sargassum Tannin, an Antibiotic Which Retards Fouling,” Nature, Vol. 208, No. 5005, 1965, pp. 52-53. http://dx.doi.org/10.1038/208052a0

[14] T. Sakami, "Effects of Algal Excreted Substances on the Respiration Activities of Epiphytic Bacteria on the Brown Alga, Eisenia bicyclis Kjellman,” Fisheries Science, Vol. 62, No. 3, 1996, pp. 394-396. http://dx.doi.org/10.2331/fishsci.62.394

[15] A. K. Swanson and L. D. Druehl, "Induction, Exudation and the UV Protective Role of Kelp Phlorotannins," Aquatic Botany, Vol. 73, No. 3, 2002, pp. 241-253. http://dx.doi.org/10.1016/S0304-3770(02)00035-9

[16] T. Shibata, K. Ishimaru, S. Kawaguchi, H. Yoshikawa and Y. Hama, "Antioxidant Activities of Phlorotannins Isolated from Japanese Laminariaceae,” Journal of Applied Phycology, Vol. 20, No. 5, 2008, pp. 705-711. http://dx.doi.org/10.1007/s10811-007-9254-8

[17] Y. Fujii, R. Tanaka, H. Miyake, Y. Tamaru, M. Ueda and T. Shibata, "Evaluation for Antioxidative Properties of Phlorotannins Isolated from the Brown Alga Eisenia bicyclis, by the H-ORAC Method," Food and Nutrition Sciences, Vol. 4, No. 8A, 2013, pp. 78-82. http://dx.doi.org/10.4236/fns.2013.48A010

[18] T. Miyasaki and K. Harada, "Feeding Attractants and Stimulants for Aquatic Animals,” Fisheries Science, Vol. 68, Suppl. 2, 2002, pp. 1406-1409.

http://dx.doi.org/10.2331/fishsci.68.sup2_1406 
[19] F. B. Whitfield, F. Helidoniotis, K. J. Shaw and D. Svoronos, "Distribution of Bromophenols in Species of Marine Algae from Eastern Australia,” Journal of Agricultural and Food Chemistry, Vol. 47, No. 6, 1999, pp. 2367-2373. http://dx.doi.org/10.1021/jf981080h

[20] H. Y. Chung, W. C. Joyce Ma, P. O. Ang Jr., J.-S. Kim and F. Chen, "Seasonal Variations of Bromophenols in Brown Algae (Padina arborescens, Sargassum siliquastrum, and Lobophora variegata) Collected in Hong Kong," Journal of Agricultural and Food Chemistry, Vol. 51, No. 9, 2003, pp. 2619-2624. http://dx.doi.org/10.1021/jf026082n
[21] Y. Agatsuma, H. Endo and K. Taniguchi, "Inhibitory Effect of 2,4-Dibromophenol and 2,4,6-Tribromophenol on Larval Survival and Metamorphosis of the Sea Urchin Strongylocentrotus nudus,” Fisheries Science, Vol. 74, No. 4, 2008, pp. 837-841. http://dx.doi.org/10.1111/j.1444-2906.2008.01596.x

[22] J.-Y. Li, H. Endo, Y. Agatsuma and K. Taniguchi, "Inhibition of Larval Survival and Metamorphosis of the Sea Urchin Strongylocentrotus intermedius by 2,4-Dibromophenol and 2,4,6-Tribromophenol," Aquaculture Science, Vol. 59, No. 2, 2011, pp. 247-253.

http://dx.doi.org/10.11233/aquaculturesci.59.247 